\title{
Failure of Administrative Data to Guide Asthma Care
}

\author{
Joel Agarwal ${ }^{1,2}$, Jennifer LaBranche ${ }^{1,2}$, Jessica Cohen ${ }^{3}$, Chris de Gara, \\ MB, MS, FRCS(Ed, Eng \& C) FACS, FFSTed ${ }^{3,4}$, Dilini Vethanayagam, \\ $M D, F^{2} C P C^{1}$
}

'Department of Medicine, University of Alberta, Edmonton, Alberta

${ }^{2}$ Department of Biological Sciences, University of Alberta, Edmonton, Alberta

${ }^{3}$ Physician Learning Program, University of Alberta, Edmonton, Alberta

${ }^{4}$ Department of Surgery, University of Alberta, Edmonton, Alberta

Corresponding author: dilini@ualberta.ca

\section{ABSTRACT}

Rationale: Asthma is a chronic inflammatory disease of the airways that is very common (7.9\% of Canadians over the age of 12). Despite numerous clinical guidelines, education events and administrative data reviews, there has been little change to the way asthma is managed in the Canadian health care system for nearly 30 years. We evaluated, through the Physician Learning Program (PLP) in Alberta, possible reasons why administrative datasets have not been able to provide meaningful information to adjust health policy.

Methods: Provincial data was attained through Alberta Health Service and Alberta Health on pulmonary function testing from 2005-2011 (through the PLP). The number of asthma diagnosis made during the same time frame were then compared.

Results: The preliminary results of the PLP found that spirometry was billed for roughly half as often as the asthma diagnostic codes were utilized during the same time frame. However, the review also revealed inconsistencies in how administrative data are captured, making it difficult to determine whether spirometry is being underutilized by physicians in making asthma diagnoses.

Conclusions: Inconsistencies in how administrative data are captured in Alberta may be contributing to an incomplete picture of the rates of asthma diagnosis and physiological testing, and may explain, in part, the limited influence of administrative datasets on guiding meaningful change within the healthcare system.

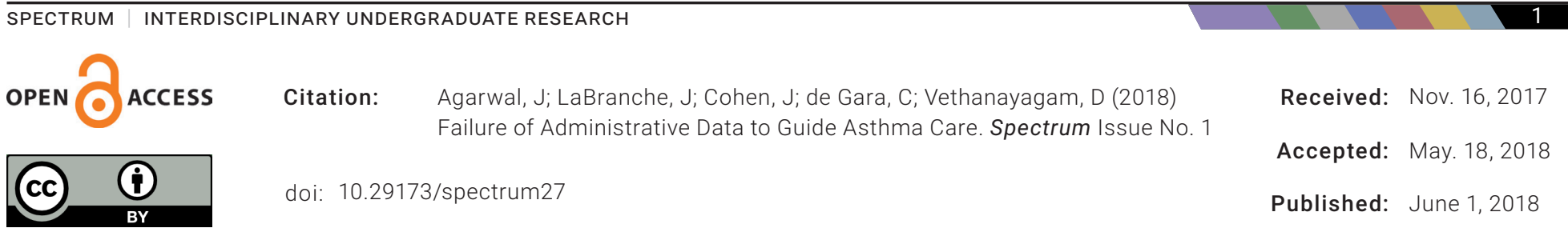




\section{Introduction}

Asthma is a prevalent disease for which numerous clinical guidelines, educational events and administrative data reviews have been implemented to guide asthma care. However, despite these resources being readily available to health care workers (HCWs) and administrators, it has not significantly changed the way in which asthma is managed in the Canadian health care system since the first Canadian guidelines were introduced in 1989. Numerous clinical guidelines and educational events have been implemented over this period; however, regional, provincial and national administrative datasets have been unable to assist health care providers $[1,2,3]$. Consequently, significant morbidity and mortality rates of asthma patients continue to cost the health care system.

The cost of respiratory diseases, excluding lung cancer, account for the fourth largest health care cost in Canada [4]. The direct costs of asthma are estimated at $\$ 600$ million per year. Asthma leads not only to direct costs from services such as physician visits, emergency visits, diagnostic tests and drug costs, but also to indirect costs from sources such as lost time from work [5] According to Statistics Canada, in 2014, 8.1\% of Canadians (2.9 million) 12 years of age and older reported having had a diagnosis of asthma from a health professional [6]. This disease accrues a mortality of 500 adults each year and is responsible for $20 \%$ of school absences in Canada [7].

Multiple publications over the past 15 years have indicated that 1 in 3 physician-diagnosed asthma cases are not in fact, asthma $[10,11]$. This includes over 700,000 Canadians who may be misdiagnosed with asthma. The diagnosis of asthma, which may become a lifelong label, should not be made in the absence of timely pulmonary physiologic testing (i.e. spirometry, pulmonary function tests [PFTs]) and airway challenge tests in conjunction with appropriate respiratory symptoms $[8,9]$. However, although the Canadian Respiratory Guidelines have outlined best practices for treatment of asthma, a Canadian study found that only $39 \%$ of physicians surveyed made treatment recommendations based on the Canadian Respiratory Guidelines [10]. A Nova Scotia study found that only $52.2 \%$ of their 90 selfreferred participants who had a diagnosis of asthma reported having received pulmonary function testing, and $62 \%$ of the participants were being treated with long-term asthma medications despite not meeting the combined clinical and physiological test criteria for a diagnosis of asthma [11].

Spirometry is a simple, cost effective test used in the diagnosis of respiratory diseases. This physiologic testing is essential in asthma diagnosis; however, it should never be used as a stand-alone diagnostic test. It must be taken into consideration after a thorough clinical pre-test likelihood is evaluated [21]. If diagnosis is made without spirometry testing, contradictory to the Canadian Respiratory Guidelines, asthma misdiagnosis may occur. Diagnosis of asthma may result in patients being prescribed medications, and if based on an incorrect diagnosis, this could be dangerous, unnecessary and costly to both the patient and the health care system. There are also side effects associated with some of the prescription medications used for asthma; for example, $\beta 2$ agonists have been found to increase cardiovascular death, and therefore pose a risk for individuals with cardiovascular disease [17]. Owing to the potential for unnecessary financial burden and health risks, it is important to ensure that an accurate diagnosis of asthma is made and managed correctly.

This study aims to evaluate possible reasons why provincial, national and territorial administrative datasets, to date, have not been able to provide meaningful solutions for this critical health care problem. 


\section{Methods}

The Physician Learning Program (PLP), an Alberta Medical Association benefit stream program, is a collaboration between the University of Alberta and University of Calgary. This program provides physicians with personal data in a manner to inform practice and modify behavior within their practice setting, an example of best practice through knowledge translation [12]

A panel of asthma experts had a project approved by the PLP steering committee (College of Physicians and Surgeons of Alberta (CPSA), Alberta Health Services (AHS) and Alberta Health $(A H))$. The project aim was to examine the question of asthma over-diagnosis and relevant data. Ethics approval was sought and obtained through the University of Alberta research ethics board. Access to administrative datasets held by AHS and AH were made available to the panel. The process carried out by the Edmonton PLP for its projects is shown in Figure 1.

Provincial asthma data on pulmonary function testing were collected from 2005-2011 to compare with the number of asthma diagnosis made during the same time frame. The project did not proceed beyond the expert panel review due to problems encountered with data.

\section{Results}

Data inconsistencies became apparent through the extraction process in terms of: (i) Physician and Pulmonary function test (PFT) lab billing; and, (ii) Data capture from public versus private PFT labs. Variance in primary care physician billings for spirometry was noted. Figure 2 shows the available respiratory testing data in Alberta, using the spirometry testing code $03.38 \mathrm{C}$ Health Service

\section{The PLP Process}

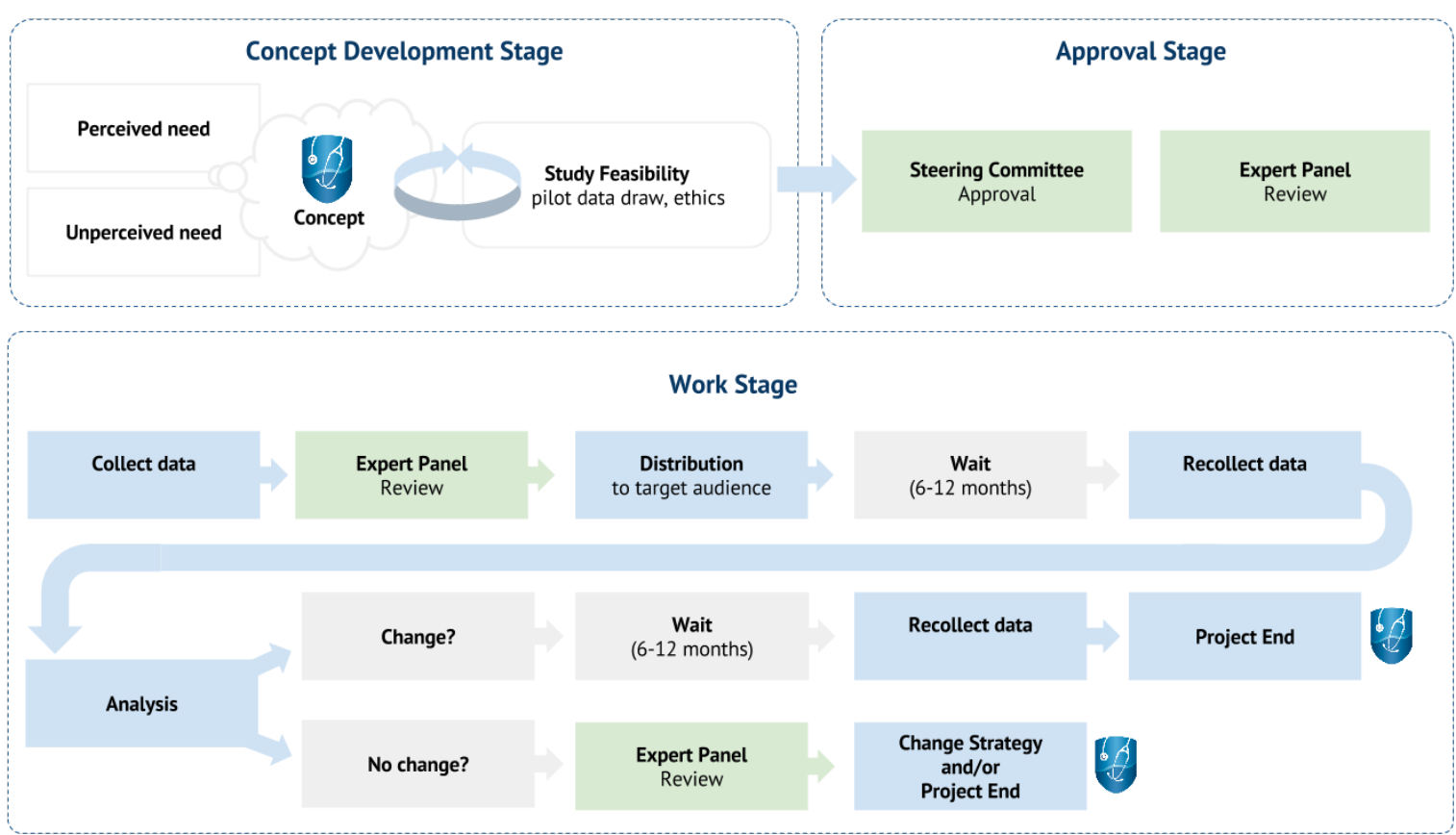

Figure 1: The PLP Edmonton project process

Image supplied by the PLP. 
Code and the asthma diagnosis Diagnostic Codes 493 (asthma), 493.0 (extrinsic asthma), 493.1 (intrinsic asthma) and 493.9 (asthma, unspecified), that were collected during three different time periods between 2005 and 2011 . The unique asthma diagnostic codes for newly diagnosed asthma were employed between 14660 and 16928 times, yet spirometry was only billed for between 6798 and 10323 times or roughly half as often as the asthma diagnostic codes.

\section{Discussion}

Our study showed that Alberta's current system to capture pulmonary physiology as assessed by the PLP is not adequate. We used provincial asthma data on pulmonary function testing performed between 2005 and 2011, along with data on asthma diagnosis made during the same time period. Due to problems with data extraction caused by inconsistencies in physician and PFT lab billing as well as differences in billing between private and public labs, the project was never completed.
Pulmonary physiology, in addition to a detailed history, is essential to the diagnosis of asthma. Asthma overdiagnosis poses health services concerns which can be impacted by pulmonary physiology testing, and where testing is done [18]. The current process for interpretation of pulmonary physiology is coordinated through the CPSA and involves four levels. To perform and report lung function tests, physicians must obtain CPSA approval as at least a level II physician, which requires a specialist in respiratory medicine, internal medicine, anesthesia or pediatrics to receive training for one month in a lab that performs 500 level III studies each year, unless it is being performed as office spirometry $[13,14]$. Physiology testing may be categorized under numerous different billing codes (i.e. vitalometry, spirometry, flow volume loops; as set out in the April 2015 Schedule of Medical Benefits explanatory codes released by Alberta Health) [15]. There are considerable inconsistencies in billing codes selected by physicians. Data capture using the spirometry billing code may underrepresent the true number of pulmonary function

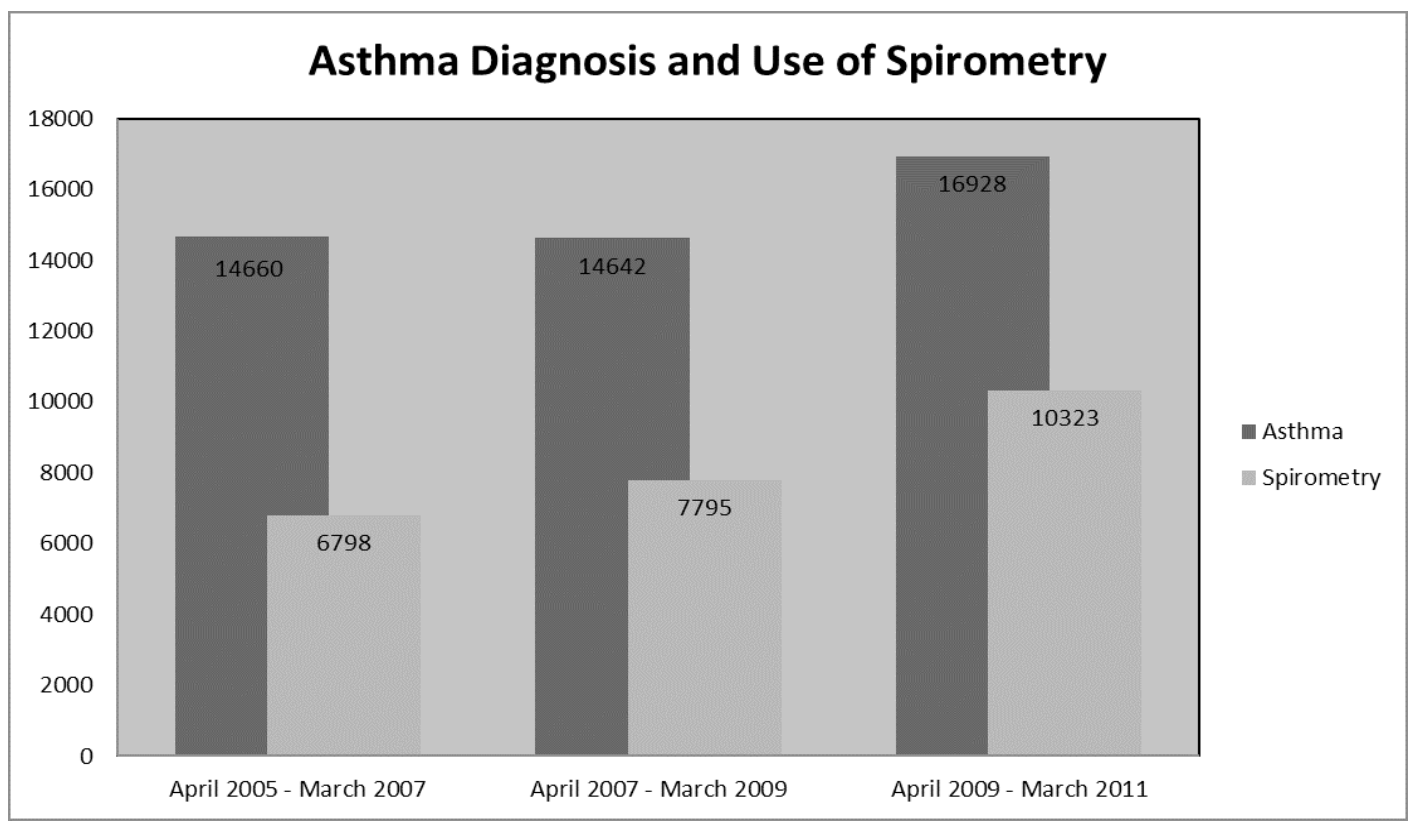

Figure 2: The initial results of the PLP respiratory testing project showing spirometry based on the $03.38 \mathrm{C} \mathrm{Health}$ Service Code and asthma diagnosis based on Diagnostic Codes 493 (asthma), 493.0 (extrinsic asthma), 493.1 (intrinsic asthma) and 493.9 (asthma, unspecified). 
tests being performed. Physicians may also bill separately for performing these tests, versus interpreting the results. They are also permitted to bill separately for each component of a test. This could lead data analysts to overestimate the number of tests being performed.

The preliminary PLP data we report here found that spirometry was billed for roughly half as often as the asthma diagnostic codes were utilized during the same time frame. The discrepancy between asthma diagnosis and use of spirometric testing is concerning, especially because of the essential nature of spirometry in asthma diagnosis. If the method of data capture for the PLP project had provided reliable data, then it could be concluded that spirometry is not consistently utilized by physicians in order to diagnose asthma, and as such these individuals may have an inaccurate and costly diagnosis.

To further compound the potential data capture errors, Alberta and other provinces have seen a profusion of private labs over recent years whose regulatory oversight may differ from provincial labs. Medical laboratory services are one of the most privatized medical services offered in Canada, and for-profit laboratories differ in several ways from public services. Firstly, forprofit laboratories tend to have less accountability because of their limited transparency, which is caused by their ability to limit disclosure of business information [16]. Secondly, for-profit laboratories pose a challenge to integration of health care information since in some provinces they fall under separate administrative structure, have different payment structure and separate data networks [16]. Potential inconsistencies in information disclosure in private labs may also contribute to knowledge gaps, and further affect the way data is managed in Alberta.

The PLP is not the first to encounter problems gathering reliable data from healthcare databases and this challenge is not unique to Alberta, or even
Canada. Silva-Costa and colleagues examined the issues associated with a central database in the Ministry of Health's Central Administration for the Health System (ACSS), which receives electronic hospital records from Portuguese hospitals daily [19]. They found major discrepancies between the data when obtained directly from the specific hospitals as opposed to when obtained from the ACSS. This could produce inaccuracies in the results of studies performed using the ACSS aggregated data [19]. A study by Scarlett et al also found inconsistencies in the Kaiser admissions database, specifically looking at ethnicity data of patients from five Northern California medical centers [20]. They compared patient selfreported ethnicity with what was documented in the Kaiser admissions database and found discrepancies, especially among individuals of Asian, Hispanic and Aboriginal descent. These raise concerns at the level of data reporting [20].

While health administrative data offer a unique source of province-wide data, on a scale not obtainable from a chart or clinic review; the usefulness of the data is limited by the discrepancies in reporting. Administrative data should be used not only as a basis for policy making, but also as a method to inform individual physician's practice. It is thus important to have data that is both accurate and relevant to a clinical setting. Through this review it has been revealed that how administrative data is captured may lead to an incomplete picture and that there may be gaps in data capture which may deter health professionals from implementing changes based on conclusions from such studies.

We have found that the current administrative system is in need of improvement to better link private for-profit PFT labs in conjunction with private not-for-profit PFT labs (i.e. AHS), into a common administrative database such that the province of Alberta has more robust data gathering capacity for quality assurance /quality control reviews related to asthma. 


\section{Acknowledgments}

This study was funded by the Physician Learning

Program.

\section{Contributions}

All authors contributed to the manuscript

development and editing, and JL compiled data

from the Physician Learning Program.

\section{Conflicts of Interest}

The authors declare that there is no conflict of interest in the publication of this article. 


\section{References}

1. Hargreave E, Dolovich J, Newhouse M. The assessment and treatment of asthma: A conference report *. J Allergy Clin Immmunol 1990; 85(6):1098-111. https://doi.org/10.1016/00916749(90)90056-A

2. Cartier A, Bernstein L, Burge PS, et al. Guidelines for Bronchoprovocation on the Investigation of Occupational Asthma - Report of the Subcommittee for Occupational Asthma on Bronchoprovocation. J Allergy Clin Immmunol 1989;84(5):823-9. https://doi.org/10.1016/00916749(89) $90346-1$

3. Lougheed MD, Lemière C, Dell SD, et al. Canadian Thoracic Society Asthma Management Continuum - 2010 Consensus Summary for children six years of age and over, and adults. Can Respir J 2010;17(1):15-24.

4. Kaplan A. Systems for the management of respiratory disease in primary care - an international series: Canada. PCRJ 2008; 17(2):73-78. https://doi.org/10.3132/pcrj.2008.00033

5. Bahadori K, Doyle-Waters M, Marra C, et al. Economic burden of asthma: a systematic review. BMC Pulm Med 2009;9:24. https://doi.org/10.1186/1471-2466-9-24

6. Statistics Canada. Asthma, by sex, provinces and territories (Percent). <http://www.statcan.gc.ca/ tables-tableaux/sum-som/l01/cst01/health50b-eng.htm> (accessed May 03, 2018).

7. The Lung Association. Asthma: A Resource for Canadian Journalists. <https://sk.lung.ca/about-us/ news-room/backgrounders-and-information-sheets/asthma-fact-sheet> (accessed May 05, 2018).

8. Coates A, Graham B, McFadden R, et al. Spirometry in Primary Care. Can Respir J 2013; 20(1): 13-22

9. FitzGerald J, Boulet L, Mclvor R, Zimmerma S, Chapman K. Asthma control in Canada remains suboptimal: The Reality of Asthma Control (TRAC) study. Can Respir J 2006; 13(5) 253-259.

10. LindenSmith J, Morrison D, Deveau C, et al. Overdiagnosis of asthma in the community. Can Respir J 2004 Mar; 11(2):111-6.

11. Aaron $\mathrm{S}$, Vandemheen $\mathrm{K}$, Boulet LP, et al. Overdiagnosis of asthma in obese and nonobese adults. CMAJ 2008 Nov 18;179(11):1121-31. https://doi.org/10.1503/cmaj.081332

12. Physician Learning Program. Physician Learning Program - Home. <http://www.albertaplp.ca/> (accessed May 3, 2018).

13. College of Physicians and Surgeons of Alberta. Qualifications to be a Medical Director of a Pulmonary Function Testing Lab. < http://www.cpsa.ca/wp-content/uploads/2017/12/PFT-LabPhysician-Qualifications.pdf> (accessed May 05, 2018).

14. College of Physicians and Surgeons of Alberta. Spirometry and Flow Volume Measurements Standards and Guidelines. <http://cpsa.ca/wp-content/uploads/2015/03/Spirometry_Flow_Volume_ Measurement_Guidelines.pdf> (accessed May 05, 2018)

15. Alberta Health. Medical Procedure List As Of 01 April 2015. <http://www.health.alberta.ca/ professionals/SOMB.html> (accessed May 05, 2018).

16. Sutherland R. The effect of for-profit laboratories on the accountability, integration, and cost of Canadian health care services. Open Medicine. 2012; 6(4).

17. Abramson MJ, Walters J, Walters EH. Adverse effects of beta-agonists: are they clinically relevant? Am J Respir Med 2003;2:287-97. https://doi.org/10.1007/BF03256657

18. Agarwal J, Saad E, Fontaine G, Pagliardini S, Dafoe W, Kherani T, Killian K, Vethanayagam D. PostBronchodilator Testing as Part of Pulmonary Physiology Laboratories: Part of the Asthma OverDiagnosis Problem? Canadian Journal of Public Health. 2018. [Submitted].

19. Silva-Costa T, Rocha-Marques B, Garcia-Lema I, Freitas A. Inconsistencies in Portuguese hospitals 
databases: Implications to hospital funding and production. IEEE 2012;1-4

20. Gomez SL, Kelsey JL, Glaser SL, Lee MM, Sidney S. Inconsistencies between Self-reported Ethnicity and Ethnicity Recorded in a Health Maintenance Organization. Ann Epidemiol. 2005;15(1):71-9. https://doi.org/10.1016/j.annepidem.2004.03.002

21. McCormack MC, Enright PL. Making the diagnosis of asthma. Respiratory Care 2008;5:583-92. 